Sādhanā Vol. 39, Part 3, June 2014, pp. 659-676. (C) Indian Academy of Sciences

\title{
Behavioural analysis of a time series- A chaotic approach
}

\section{T A FATHIMA and V JOTHIPRAKASH*}

Department of Civil Engineering, Indian Institute of Technology Bombay, Mumbai 400 076, India

e-mail: vprakash@iitb.ac.in; tafathima@gmail.com

MS received 31 October 2013; revised 15 January 2014; accepted 20 January 2014

\begin{abstract}
Out of the various methods available to study the chaotic behaviour, correlation dimension method (CDM) derived from Grassberger-Procaccia algorithm and False Nearest Neighbour method (FNN) are widely used. It is aimed to study the adaptability of those techniques for Indian rainfall data that is dominated by monsoon. In the present study, five sets of time series data are analyzed using correlation dimension method (CDM) based upon Grassberger-Procaccia algorithm for studying their behaviour. In order to confirm the results arrived from correlation dimension method, FNN and phase randomisation method is also applied to the time series used in the present study to fix the optimum embedding dimension. First series is a deterministic natural number series, the next two series are random number series with two types of distributions; one is uniform and another is normal distributed random number series. The fourth series is Henon data, an erratic data generated from a deterministic non linear equation (classified as chaotic series). After checking the applicability of correlation dimension method for deterministic, stochastic and chaotic data (known series) the method is applied to a rainfall time series observed at Koyna station, Maharashtra, India for its behavioural study. The results obtained from the chaotic analysis revealed that CDM is an efficient method for behavioural study of a time series. It also provides first hand information on the number of dimensions to be considered for time series prediction modelling. The CDM applied to real life rainfall data brings out the nature of rainfall at Koyna station as chaotic. For the rainfall data, CDM resulted in a minimum correlation dimension of one and optimum dimension as five. FNN method also resulted in five dimensions for the rainfall data. The behaviour of the rainfall time series is further confirmed by phase randomisation technique also. The surrogate data derived from randomisation gives entirely different results when compared to the other two techniques used in the present study (CDM and FNN) which confirms the behaviour of rainfall as chaotic. It is also seen that CDM is underestimating the correlation dimension, may be due to higher percentage of zero values in rainfall data. Thus, one should appropriately check the adaptability of CDM for time series having longer zero values.
\end{abstract}

*For correspondence 
Keywords. Chaos; correlation dimension method; false nearest neighbour method; phase randomization; embedding dimension; correlation exponent; correlation dimension; daily rainfall data; India.

\section{Introduction}

In modern hydrological studies, large importance is given to time series modelling that are further used for design, planning, and operation of water resources systems. Time series represents a quantitative measure of a physical process ' $x_{t}$ ' recorded at time ' $t$ ' which can be discrete or continuous. Mostly, these observations are made at equally spaced, discrete time intervals (Brockwell \& Davis 2002). It is worth mentioning that all hydrological processes are considered to be continuous for modelling even though they are measured at discrete regular time intervals. The observation made upon a single variable of a process forms a univariate time series. A basic assumption in hydrological time series analysis or modeling is that some aspects of the past pattern will continue to remain in the future (Ramasubramanian 2007). In other ways, prediction of future values in a time series can be carried out using past values of the variable under study. Thus, time series modelling is generally not dependant on other variables and hence it is comparatively easier when the historical time series data of a process is readily available (Chatfield 1996). Time series analysis provides a compact description of the observed data, which can be used in modelling to predict future values (Harvey 1981). It also helps to determine the behaviour of the time series, which further helps in modeling the time series (Brockwell \& Davis 2002).

The behaviour of a time series in hydrology is usually considered as deterministic or stochastic for modelling purpose (Hipel \& McLeod 1994). Deterministic model assumes that the output is certain if the input is fixed and hence performance of deterministic models can fail in a dynamic system. Simple deterministic models has been developed by statistical methods like linear regression and nonlinear curve fitting techniques (Mitasova \& Mitas 1998; Jothiprakash et al 2007; Magar \& Jothiprakash 2011). In such cases where the influence of unknown factors is sizable, accurate prediction is not possible, but within a known confidence interval it may be possible and this is called a stochastic (or probabilistic) process or 'Random walk' process (Adhikari \& Agrawal 2013). In case of stochastic models, there will be a random component that has different outputs for a unique input for various model runs. Usually differencing and autoregressive models are used for predicting such series.

Three broad classes of linear stochastic models are the autoregressive (AR) models, the moving average (MA) models and the integrated (I) model (Box \& Jenkins 1976). These three classes depend linearly on previous data points. Combinations of these models produce autoregressive moving average (ARMA) and autoregressive integrated moving average (ARIMA) models. Linear stochastic methods, especially ARIMA of time-series analysis, modelling and forecasting (Box \& Jenkins 1976) have been widely used in hydrologic time-series forecasting. But these stochastic models work under the assumption of stationary and linearity. Box \& Jenkins (1976) were the pioneers of developing methodologies for univariate time series modelling based on the concepts of probability and statistics. According to Box et al (1994) an observed time series can be decomposed into four components such as original data, trend component, seasonal component and an error component which helps to find the pattern in the time series for forecasting. Detailed discussion regarding ARIMA modelling along with several case studies can be found in Pankratz (1983). Tong (1990) developed a non-linear time-series model for a dynamic system. As every model has got its own merits and limitations, the selection of an appropriate model is difficult and hence there is a scope for further improvement (Jothiprakash \& Magar 2009). 
Thus, in the recent past, the subject of time series analysis has been evolving at an astonishing rate. However, in the case of deterministic and stochastic modelling (ARIMA) nonlinear behaviour of time series is not taken into account. Over the past two decades, researchers have accepted nonlinear approach as a viable alternative. The nonlinear approach includes nonlinear stochastic methods (Govindaraju 2002; Kavvas 2003) and artificial neural networks (Govindaraju \& Rao 2000; ASCE Task Committee 2000a, b).

Artificial Intelligence (AI) based data driven techniques such as artificial neural networks (ANN), adaptive neuro-fuzzy inference system (ANFIS), linear genetic programming (LGP) and Model tree (MT) have emerged as important tools to overcome the drawbacks of conventional modelling technique (Zhang 1998; Hamzacebi 2008; Kumar 2004; Jothiprakash \& Magar 2012). These data driven approaches are based on extracting and re-using information available within the hydrological time-series (De Vos \& Rientjes 2005). These tools and techniques are more flexible and can be used for modelling the systems on a real-time basis. In the last decade, ANN's have been successfully used in hydrological modelling to model non-linear system efficiently (Tokar \& Johnson 1999; Thirumalaiah \& Deo 2000; Chang et al 2002; Sivakumar et al 2002; Wu et al 2005; Jothiprakash \& Magar 2012). However, ANN has some limitations like the difficulty in choosing the appropriate training algorithm, time-consuming effort involved in developing the structure and difficulty in extracting the knowledge from ANN model. The major drawbacks in ANN are finalizing the appropriate architecture and also in extracting the knowledge gained for dissemination to field engineers. On the other hand the recent techniques like MT and LGP are overcoming this disadvantage. Non-linear relationship can be easily mapped with simple LGP model than other time consuming data driven techniques (Khu et al 2001; Brameier \& Banzhaf 2007; Guven 2009; Garg \& Jothiprakash 2010). MT is another promising technique (Quinlan 1992) which is easy to understand compared to ANN, also the relationship between input and output is comprehensible (Jothiprakash \& Kote 2011). MT is advantageous in accurate prediction and compactness of the results and also it exploits the local linearity of data. MT also found to be more advantageous than ANN as it can predict the value greater than the observed data by extrapolation which is not possible in case of ANN (Solomatine \& Dulal 2003; Pal \& Deswal 2009; Jothiprakash \& Kote 2011).

Even though AI models consider nonlinearity of the system to predict the hydrological processes, it still requires identification of number of variable (dimension) which governs the future values; proper selection of model orders, the number of input, hidden and output neurons is crucial for a successful forecasting (Adhikari \& Agrawal 2013). The number of input variables is usually identified by trial and error method which is computationally difficult. An approach that can couple deterministic and stochastic approach involving the non linearity may be a better choice. The idea of deterministic dynamical systems and chaos played a key role in the unification of ideas concerning nonlinear modelling by placing them in the framework of dynamical hydrological systems. Chaos theory can offer such a coupled deterministic stochastic approach, since its underlying concepts of nonlinear interdependence, hidden determinism and order, sensitivity to initial conditions are highly relevant in hydrology (Sivakumar et al 2007).

Several nonlinear methods, such as: method of time delays (Takens 1981), correlation dimension (Grassberger \& Procaccia 1983a, b), Lyapunov spectrum (Sano \& Sawada 1985), mutual information (Fraser \& Swinney 1986), False nearest neighbour method (Kennel et al 1992), Cao's method (Cao 1997), have been used for identifying the behaviour and to analyse the properties of the systems. It is reported that the number of dimensions that governs a particular process can be identified through nonlinear chaotic analysis which is computationally easier (Sivakumar 2000). 
The chaos theory says that random input is not the only reason for irregularity of a system, but a nonlinear chaotic system can behave randomly even if the underlying dynamics is governed by purely deterministic equations (Kantz \& Schreiber 2003). The randomness in most of the natural systems can be attributed to this reason. But because of the lack of data and computational power, most of the analysis and prediction techniques used linear stochastic approach (Harms \& Campbell 1967; Klemes 1978; Salas \& Smith 1981).

Analysing the data for chaotic behaviour can reveal whether an erratic looking time series is actually deterministic, leading to more accurate short-term predictions. It also indicates whether long term predicting for the system is feasible or not. Thus simplifies the modelling effort and produces more accurate prediction. The chaos theory has made revised concepts about determinism versus randomness, in nonlinear analysis (Williams 1997).

Fairly large number of deterministic, stochastic and artificial intelligence models are available but researchers are seeking better models for accurate prediction of hydrological processes especially peak value. In these AI models, identification of variable (dimension) which governs the future values is either by correlation studies (ACF, PACF and cross correlation) or by trial and error processes as in ANN and other data driven models (Chang et al 2002; Sivakumar et al 2002). It is reported that the number of dimensions that governs a particular process can be identified through non linear chaotic analysis (Sivakumar 2001). But, chaotic analysis may not reveal underlying physical laws (Williams 1997). Fairly a good number of works has been reported on application of chaotic studies for many hydrological processes like rainfall (Sivakumar et al 1998) and other parameters (Sivakumar \& Jayawardena 2002). There are many tools and techniques available for the analysis of behaviour of a time series. Most widely used method, correlation dimension method developed by Grassberger \& Procaccia (1983a, b) is one among them and is considered for the present study to analyse the behaviour of a time series. The algorithm helps to classify the time series into a deterministic, stochastic or chaotic series. The correlation dimension method based upon Grassberger-Procaccia algorithm has been widely used for behavioural study in hydrology and identification of number of dominant variables of the system (Hense 1987; Jayawardena \& Lai 1994; Porporato \& Ridolfi 1997; Sivakumar et al 1998; Kantz \& Schreiber 1997; Islam \& Sivakumar 2002; Khatibi et al 2011; Dhanya \& Kumar 2011; Jothiprakash \& Fathima 2013a, b).

The main objective of the study is to prove that the correlation dimension method based on Grassberger-Procaccia algorithm is a robust method and can predict the behaviour of a time series accurately. For such a study initially four sets of time series with known behaviour namely natural number series, uniform distributed random number series, normal distributed random number series, and Henon data is analysed using Grassberger-Procaccia algorithm. By analyzing these known series it is easy to find the reliability of Grassberger-Procaccia algorithm for real life data analysis. As a case study, the method is then applied to a real life daily rainfall data observed at Koyna rain gauge station, Maharashtra, India.

\section{Methodology}

In the present study, Correlation dimension method (CDM) based on Grassberger-Procaccia algorithm and False nearest neighbour method (FNN) are used to determine the chaotic behaviour of the time series. Initially correlation dimension method is applied to the time series for its behavioural study. To confirm the results arrived from Correlation dimension method, False nearest neighbour method is then applied to all the time series used in the present study to fix the optimum embedding dimension for modelling. Correlation dimension is a measure of 
extent to which the presence of data points with respect to other points lying on the attractor in phase space. Initially the non linear chaotic analysis is applied to the deterministic time series, then to random number series and finally applied to chaotic series to reveal the reliability of Grassberger -Procaccia algorithm. Delay time has to be fixed for finding the correlation integral. In the present study delay time is chosen as one which is same as the sampling frequency of data. Considering the given data as basic series $\mathrm{Y}_{t}$, the subseries are obtained by lagging the data with delay time as lag. The dynamics of time series $\left\{\mathrm{x}_{1}, \mathrm{x}_{2}, \ldots \mathrm{x}_{n}\right\}$ are fully captured or embedded in the m-dimensional phase space (assuming delay time as 1 ) defined by eq. 1 .

$$
\mathrm{Y}_{\mathrm{t}}=\left\{\mathrm{x}_{\mathrm{t}}, \mathrm{x}_{\mathrm{t}-1}, \mathrm{x}_{\mathrm{t}-2}, \ldots \mathrm{x}_{\mathrm{t}-(\mathrm{m}-1)}\right\}
$$

The procedure usually begins by embedding the data (basic series and subseries) in a twodimensional pseudo phase space. For a given radius ' $r$ ', count the number of points within distance ' $r$ ' from the reference point. The step in radius has to be chosen depending on the magnitude of the data in time series. The only condition for selecting the initial value of ' $r$ ' is that the number of steps between initial correlation integral to final correlation integral which is equal to one (when all the points comes inside the circle for a higher range of ' $r$ ') should be large enough to fix the scaling region. After fixing the initial radius ' $r_{1}$ ' count the number of points coming inside a circle with centre as first point in phase space and radius ' $r$ '. With the same radius count the number of points coming inside the circle with centre as second point on phase space and so on. Finally the sum of all the counts for the initial radius ' $r_{1}$ ' gives the correlation sum which has to be normalised to obtain the correlation integral. The next step is to increase the radius by constant interval (say ' $r_{2}$ ') and find correlation integral using the same procedure. The radius should be increased to a particular level in constant interval such a way that all points in the phase space diagram should come under that circle. In that case correlation integral will be one. Then find correlation integral $(\mathrm{Cr}$ ) values for different values of ' $r$ ' by increasing steps in constant intervals by using the following eq. 2 (Sivakumar 2001). The whole procedure has to be repeated by increasing embedding dimensions.

$$
\mathrm{C}(\mathrm{r})=\lim _{n \rightarrow \infty} \frac{2}{\mathrm{~N}(\mathrm{~N}-1)} \sum_{\mathrm{i}, \mathrm{j}(1<\mathrm{i}<\mathrm{j}<\mathrm{N})} \mathrm{H}(\mathrm{r}-|\mathrm{Yi}-\mathrm{Yj}|)
$$

where $\mathrm{H}$ is the Heaviside step function, with $\mathrm{H}(\mathrm{u})=1$ for $\mathrm{u}>0$, and $\mathrm{H}(\mathrm{u})=0$ for $u \leq 0$, $\mathrm{N}=$ Number of data points

$$
\mathrm{u}=r-|Y i-Y j|
$$

' $r$ ' is the radius of the sphere centred on $\mathrm{Y}_{i}$ or $\mathrm{Y}_{j}$

$Y_{i}$ is the basic series of rainfall data

$Y_{j}$ is the subseries of rainfall data.

The correlation integral for various ' $r$ ' steps is plotted on a logarithmic sheet to find the correlation exponent (slope of the scaling region of $\log \mathrm{r} \mathrm{Vs} \log \mathrm{Cr}$ plot). The scaling region is the most linear portion of $\log \mathrm{r} \mathrm{V} \log \mathrm{Cr}$ plot. By plotting correlation exponent on a graph sheet for various embedding dimensions, the behaviour of the time series can be studied well. If the correlation dimension is constant for all ' $m$ ' the time series will be deterministic and if the correlation exponent increases with increase in ' $m$ ' the time series will be stochastic. If the latter relation seems to become asymptotic, the time series represents a chaotic nature. If it is chaotic, the next nearest whole number value of saturation of correlation exponent $(v)$ gives the 'correlation dimension' for the time-series. The number of variables required for modelling will be either the nearest 
integer of saturating value of correlation exponent or the dimension at which correlation exponents saturates (optimum embedding dimension) which ever is higher. Correlation dimension or optimum embedding dimension value gives the number of dimensions required to predict the future value.

The false nearest neighbours (FNN) algorithm is used as a method for determining the proper embedding dimension for recreating or unfolding the dynamics of nonlinear systems (Kennel et al 1992; Abarbanel et al 1993). The FNN algorithm is based on the geometry of the phase space reconstructed using a single time series. If there is enough information in the reconstructed phase space to predict the future output, then two vectors which are close in the reconstructed phase space will also have future outputs which are close. If the embedding is not done in enough dimensions, then there will be some neighbours in the space with vastly different outputs even though their trajectories are close in the phase space. Such neighbours are called false neighbours, since they are close to each other spatially, because of projection into a space with a dimension too small to represent the dynamics of the system. In order to find whether the neighbours are true or false, a ratio test $\left(\mathrm{R}_{k}\right)$ is conducted to determine whether the distance between the future values are significantly larger than the distance between the neighbours which are close in reconstructed phase space.

In FNN method, for each point in the phase space, $\mathrm{Y}_{k}$, its nearest neighbours $\left(\mathrm{Y}_{j}\right)$ are searched in the ' $\mathrm{m}$ ' dimensional phase space. The Euclidean distance between $\mathrm{Y}_{m}(\mathrm{k})$ and $\mathrm{Y}_{m}(\mathrm{j})$ is calculated. Then their Euclidean distance in $(\mathrm{m}+1)^{t h}$ dimension is found out and compute the value of $\mathrm{R}_{k}$ as given in eq.3.

$$
R_{m}(k)=\frac{\left\|Y_{m}(k+1)-Y_{m}(j+1)\right\|}{\left\|Y_{m}(k)-Y_{m}(j)\right\|} .
$$

If the ratio between these two distances are greater than a threshold value, $\mathrm{R}_{t}$, then the neighbours are considered to be true or vice versa. This is done for each and every point in phase space and percentage of points in data set which has false neighbours is found. This algorithm is repeated for higher ' $m$ ' value until the percentage of false neighbours drops to zero or some acceptably small number. Then the dimension of the reconstructed vector is said to be large enough to make accurate prediction of future outputs. The FNN method can also distinguish chaos from noise. Until the proper embedding is reached, the fraction of false neighbours will be high and it decreases gradually.

Phase randomisation is another technique used to confirm the behaviour of rainfall as chaotic in the present study. The data generated after randomisation is called the surrogate data (Theiler et al 1992). If the correlation dimension obtained from the surrogate data is significantly different from that of the original series, then the null hypothesis of the original time series emanated from a linear process is rejected. On the other hand, if the correlation dimension from the surrogate data and the original series are not significantly different, then the original time series is considered to be a linear stochastic process. By applying correlation dimension method on this surrogate data, the estimated correlation dimension will be invariant, since, the convergence of the dimension is forced by the shape of the power spectrum and not due to any low dimensional dynamics (Dhanya \& Nagesh Kumar 2010).

\section{Sample time series used in the present study}

Four set of time series each having a length of 10,000 data are considered for their behavioural study. The behaviour of the first three series having 2000 data points has been analysed for their behaviour by Jothiprakash \& Fathima (2013a). First series is a deterministic natural number 
series (numbers from 1 to 10,000), the next two series are random number series with two types of distributions, one is uniform (numbers between 0 to1), and another is normal distributed random number series (numbers between $-1-+1$ ). Ten thousand natural numbers, uniform random numbers, and normal random numbers are generated using Microsoft Office Excel. The fourth series is Henon data, an erratic data generated from a deterministic non linear equation.

Henon data is the most studied dynamical system which exhibits chaotic behaviour. This well known chaotic data is generated using the eq. 4 . The usual values for ' $a$ ' and ' $b$ ' which produces chaotic sequence from this equation is at $\mathrm{a}=1.4, \mathrm{~b}=3$ and the initial values of ' $\mathrm{x}_{n-1}$ ' and ' $\mathrm{x}_{n}$ ' is assumed as $\mathrm{x}_{n-1}=\mathrm{x}_{n}=1$.

$$
x_{n+1}=a+b x_{n-1}-x_{n}^{2}
$$

All the four set of data are generated continuously and hence are considered as time series for the present study with time ' $t$ ' (considered as sequential order) varies from 1 to 10,000. The statistics of these four sets of data is given in table 1. The applicability of a Grassberger-Procaccia algorithm is verified by applying it to these four series.

The time series plots of the four series with known behaviour used in the present study is shown in figure 1. Figure 1 reveals that all the time series looks erratic in nature except the first one (natural numbers series). In figure 1a, it appears to be passing through origin as there are large numbers of data (even though it is not passing through origin as natural number starts from 1). Hence, the portion near origin is magnified and depicted along with figure 1a. It is difficult to identify the behaviour of time series from the time series plot. In the present case, the uniform random numbers and Henon data pattern looks alike even though they come under different category.

After checking the applicability of correlation dimension method for deterministic, stochastic and chaotic data, the method is then applied to real life daily rainfall data observed at Koyna rain gauge station, Maharashtra, India. The statistical properties of the observed daily rainfall data are also presented in table 1 . Forty seven years of daily data are considered for the study out of which $70 \%$ of data (11969 data points) is zero as the rainfall happens only in 5 months from June to October.

Table 1. Statistics of the time series used in the present study for chaotic analysis.

\begin{tabular}{lccccc}
\hline Statistics & Natural numbers & UDR & NDR & Henon data & Rainfall \\
\hline No. of data & 10000 & 10000 & 10000 & 10000 & 17166 \\
Maximum & 10000 & 1 & 3.85 & 1.27 & 558 \\
Minimum & 1 & 0 & -3.51 & -1.28 & 0 \\
Average & 5000.50 & 0.49 & 0.00 & 0.26 & 13.32 \\
Standard deviation & 2886.90 & 0.29 & 0.99 & 0.72 & 35.28 \\
Coefficient of variation & 0.58 & 0.58 & $\alpha$ & 2.82 & 2.64 \\
Skewness & 0 & 0.02 & 0.01 & -0.50 & 4.25 \\
Kurtosis & -1.20 & -1.19 & -0.06 & -0.87 & 24.59 \\
No. of zeros & 0 & 0 & 0 & 0 & 11969 \\
Percentage of zeros & 0 & 0 & 0 & 0 & 69.73 \\
\hline
\end{tabular}

*UDR-Random number generated using uniform distribution

*NDR-Random number generated using normal distribution 

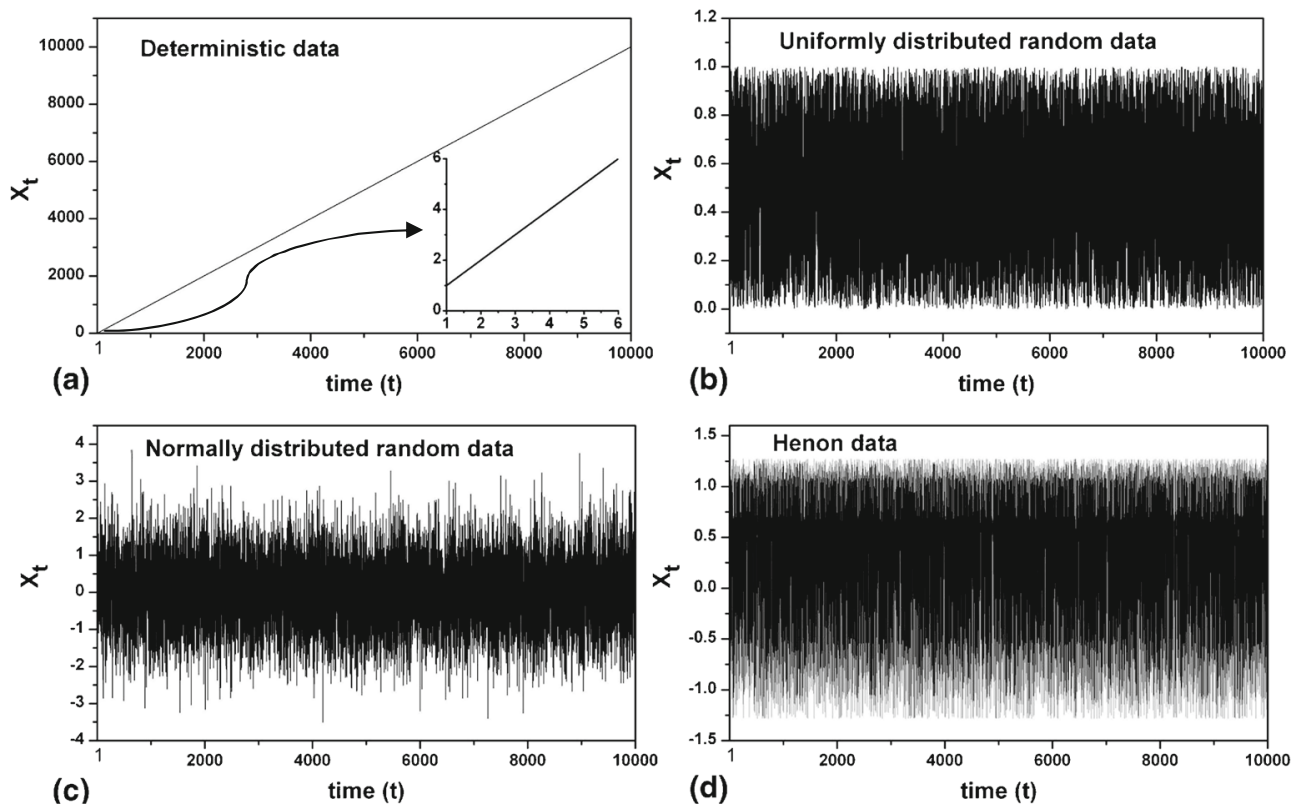

Figure 1. Time series plot of the generated data with known behaviour.

\section{Results and discussions}

Initially the time series data with known behaviour is analysed using Grassberger-Procaccia algorithm and the results are discussed below. The phase space plot, log r vs. log Cr plot and embedding dimension vs. correlation exponent plots, that are used to carry out the behaviour analysis of a time series are presented in this section. Phase space reconstruction helps to confine the data into a small space which further helps to calculate the correlation integral. The correlation integral plot helps to identify correlation exponents from scaling region for various embedding dimensions. The correlation exponent plot with various embedding dimension finally confirms the behaviour of the time series. Finally, the analysis using correlation dimension method is applied for a real life daily rainfall data at Koyna, Maharashtra, India and the results are also presented.

For deterministic series, initial value of ' $r_{1}$ ' is taken as 200 , for uniformly distributed random number ' $r_{1}$ ' is taken as 0.01 whereas for normal distributed random numbers it is 0.05 and that for Henon data is 0.03 in the present study. In the present study, embedding dimension has been increased from 1 to 20 dimensions to find the correlation integral. The delay time $(\tau)$ considered for all the five data sets is one which is the sampling frequency of each data itself.

\subsection{Phase space reconstruction}

A standard phase space is the graph between different kinds of variables. But in chaos theory mostly pseudo phase space diagram is used for determining behavioural study (Williams 1997; Strogatz 1994). The two dimensional phase space diagram of natural numbers, uniform random numbers, normal random numbers, and Henon data are given in figure 2. The phase space pattern differs from one another in the present study. A good attractor is the initial indication of chaos 
which is clear in case of Henon data (figure 2d). Even though uniform and normal random numbers comes under the same category in terms of behaviour, the phase space plot is entirely different and hence it is difficult to confirm the exact behaviour of the time series from the phase space plot. To differentiate between phase space and scatter plot, scatter plot of the four series (natural number series, uniform random series, normal random series, Henon data series) having delay time $(\tau=1)$ is plotted in figures $3 \mathrm{a}$ to $3 \mathrm{~d}$. It can be seen that they are one and the same except that lagged points are connected in phase space to study the trajectory of the points.

\subsection{Correlation integral plot on logarithmic scale}

The correlation integral for various ' $r$ ' values ranging from ' $r_{1}$ ' to ' $r_{n}$ ' for various embedding dimension ranging from $\mathrm{m}=1$ to $\mathrm{m}=20$ is plotted and shown in figure 4. More or less confirmation about the behaviour can be anticipated from $\log r$ vs. $\log \mathrm{Cr}$ plot. If the time series data is having deterministic behaviour the plots remains parallel to each other for all embedding dimensions as in case of figure $4 \mathrm{a}$. On the other hand for a stochastic time series, the spread of the plot will be more and the lines are not parallel to each other as seen in figures $4 \mathrm{~b}$ and $\mathrm{c}$. The correlation integral plot for Henon data is showing a mixed behaviour of deterministic and stochastic series. For smaller range of ' $m$ ' the behaviour is more or less same as that of stochastic series (lines are not parallel to each other and are spread from one embedding dimension to other) and at higher dimension, the behaviour is same as that of deterministic plot (lines are parallel to each other). For lower range of ' $r$ ' irrespective of embedding dimension the behaviour is
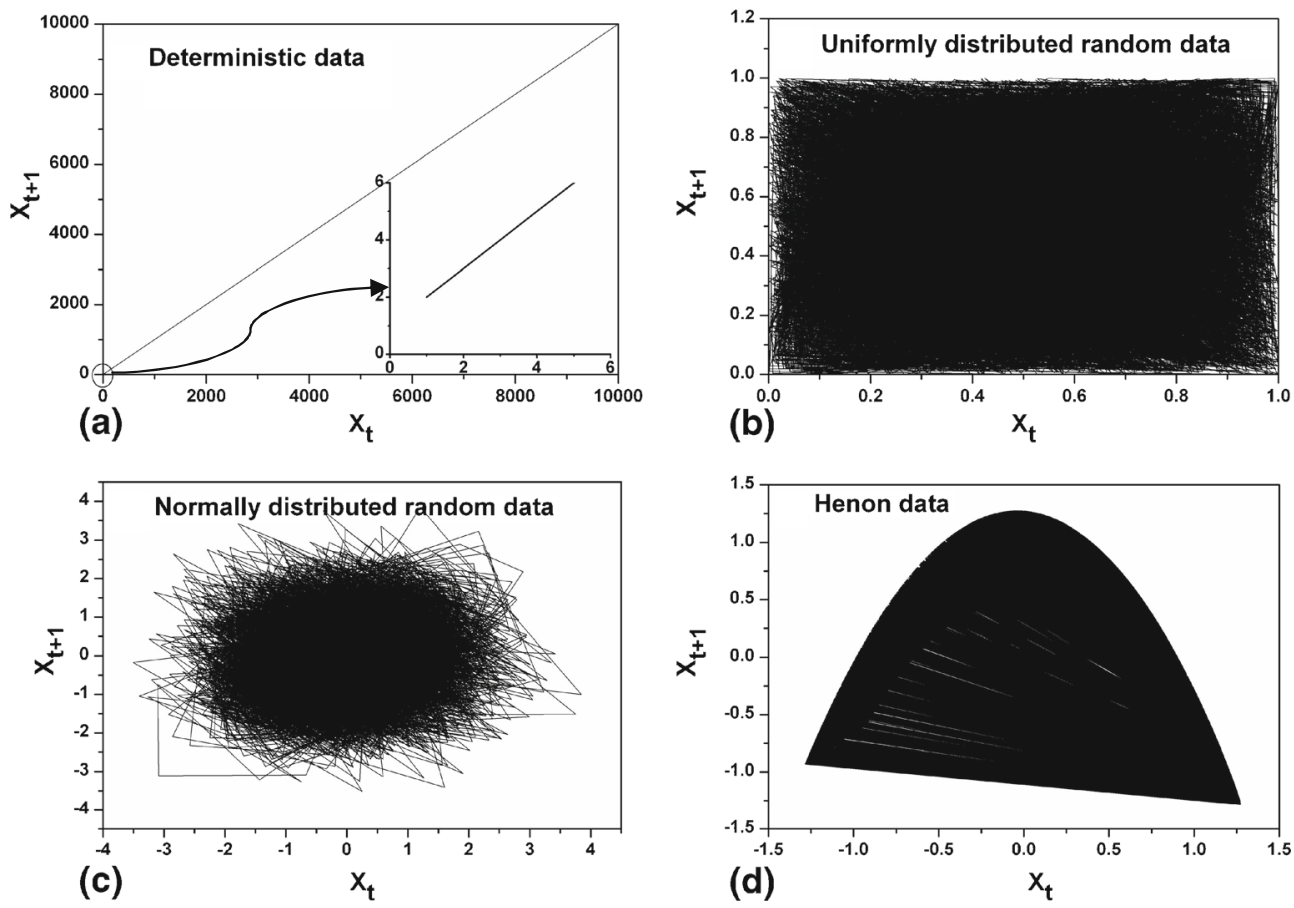

Figure 2. Phase space plots of the generated series. 

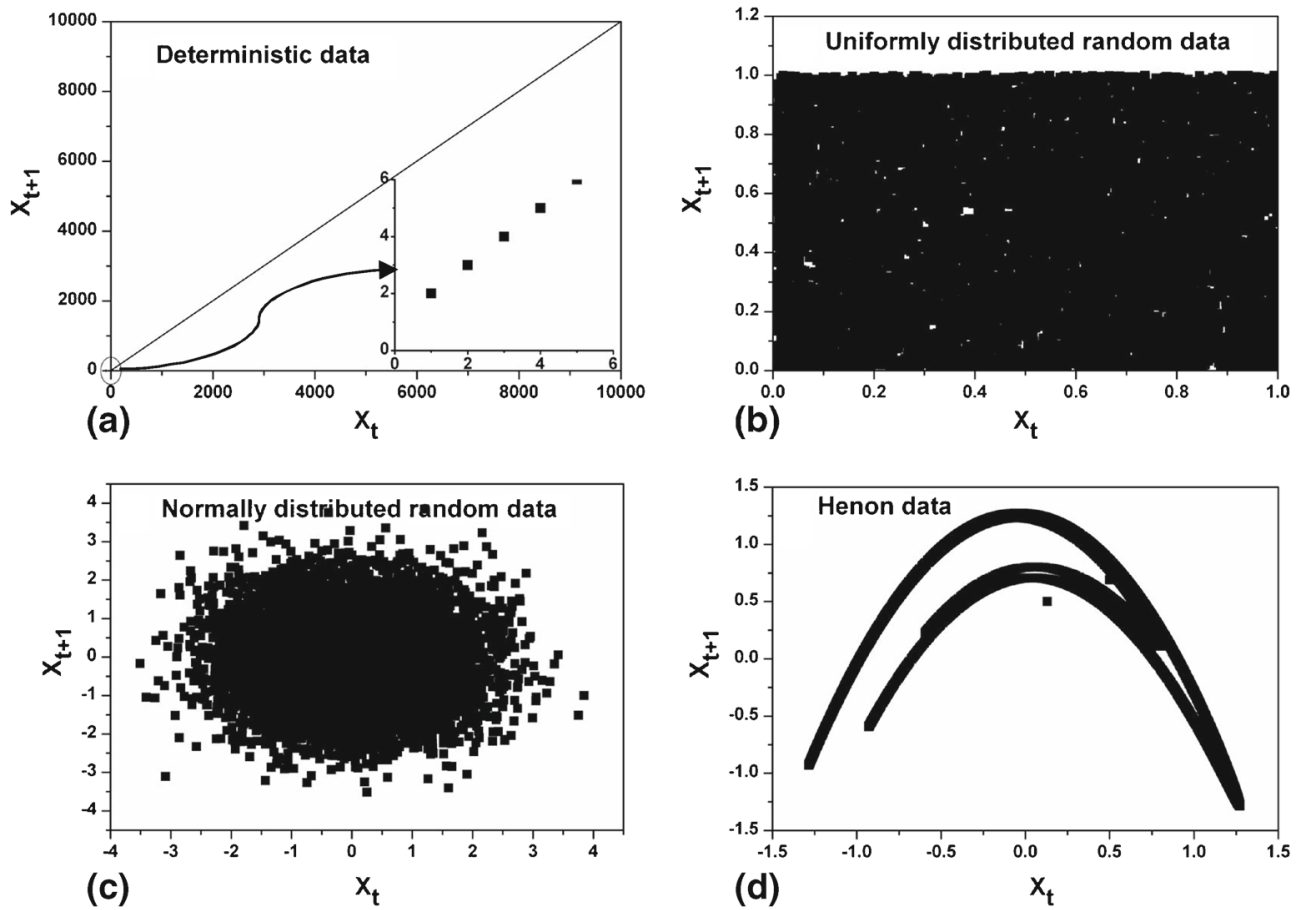

Figure 3. Scatter plot of the generated series.

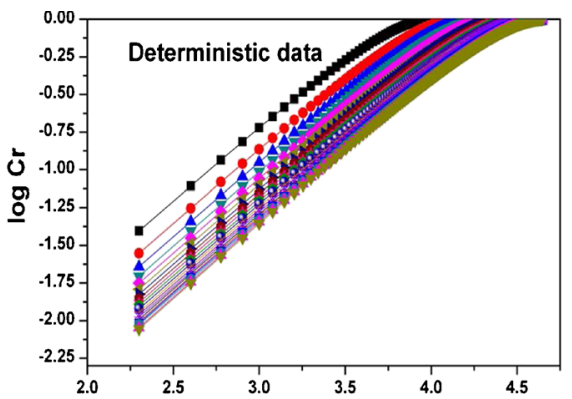

(a)
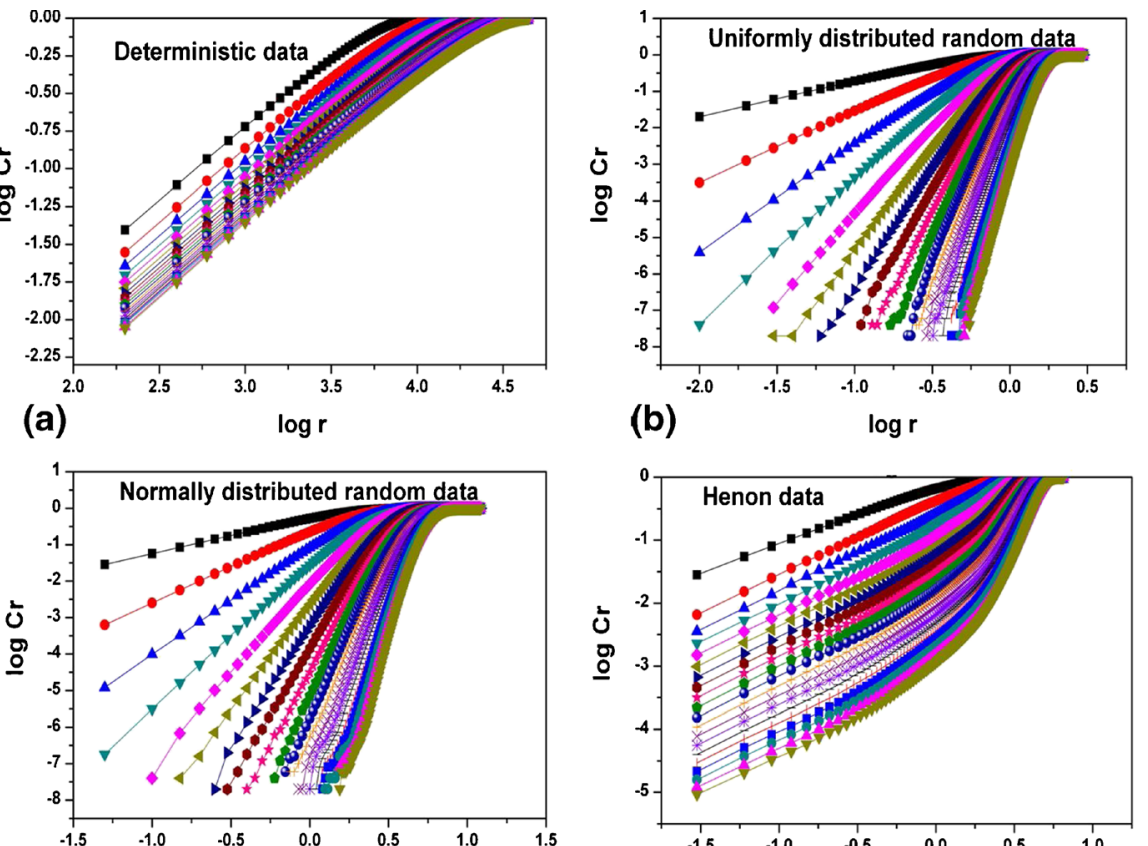

(c)

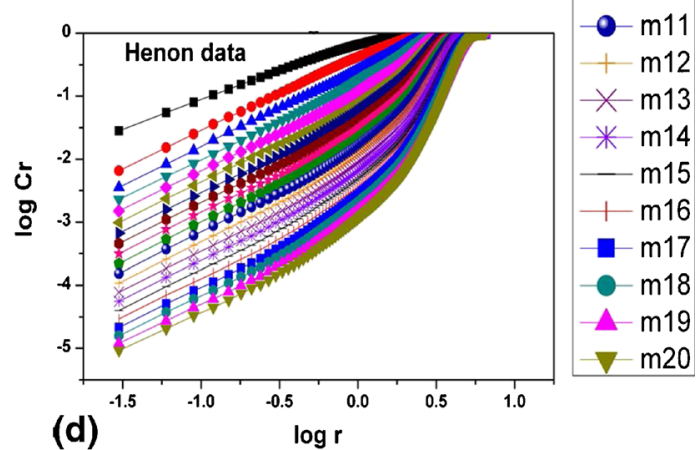

Figure 4. Correlation integral plot of the time series under behavioural study (log r vs. $\log$ Cr plot). 
linear and scaling region is more prominent (region to find the slope of the line). But the scaling region is almost absent for higher range of ' $r$ ' as there is non- linearity in the relationship between $\log \mathrm{r}$ vs. $\log \mathrm{Cr}$.

\subsection{Correlation exponent plot for various embedding dimensions}

Even though the behaviour of time series can be studied from $\log \mathrm{r}$ vs. $\log \mathrm{Cr}$ plot, the number of dominant variables controlling the system (dimensions required for predicting future value) is not clear from $\log \mathrm{r}$ vs. $\log \mathrm{Cr}$ plot. To estimate the number of dimensions required for prediction models and also to confirm the behaviour the time series, correlation exponents are calculated from $\log \mathrm{r}$ vs. $\log \mathrm{Cr}$ plot and it is plotted against various embedding dimensions. The correlation exponents are the slopes estimated from the most linear portion of the correlation integral graph plotted on logarithmic scale (Shivamoggi 1997; Sivakumar 2000). This region is the scaling region of the correlation integral plot. Thus, there will be a unique correlation exponent (slope) for each embedding dimension. The embedding dimension vs. correlation exponent plot for all the four sets of data are given in figure 5 which further clarifies the behaviour of each time series.

It is seen that the correlation exponent remains constant for all embedding dimension in case of natural numbers proves its deterministic nature from correlation exponent plot (refer figure 5a). The increasing trend of correlation exponents with embedding dimension is a stochastic nature (refer figures $5 \mathrm{~b}$ and c). Hence, it is concluded that the Grassberger-Procaccia algorithm exactly finds the behaviour of stochastic time series irrespective of its method of generation/distribution.
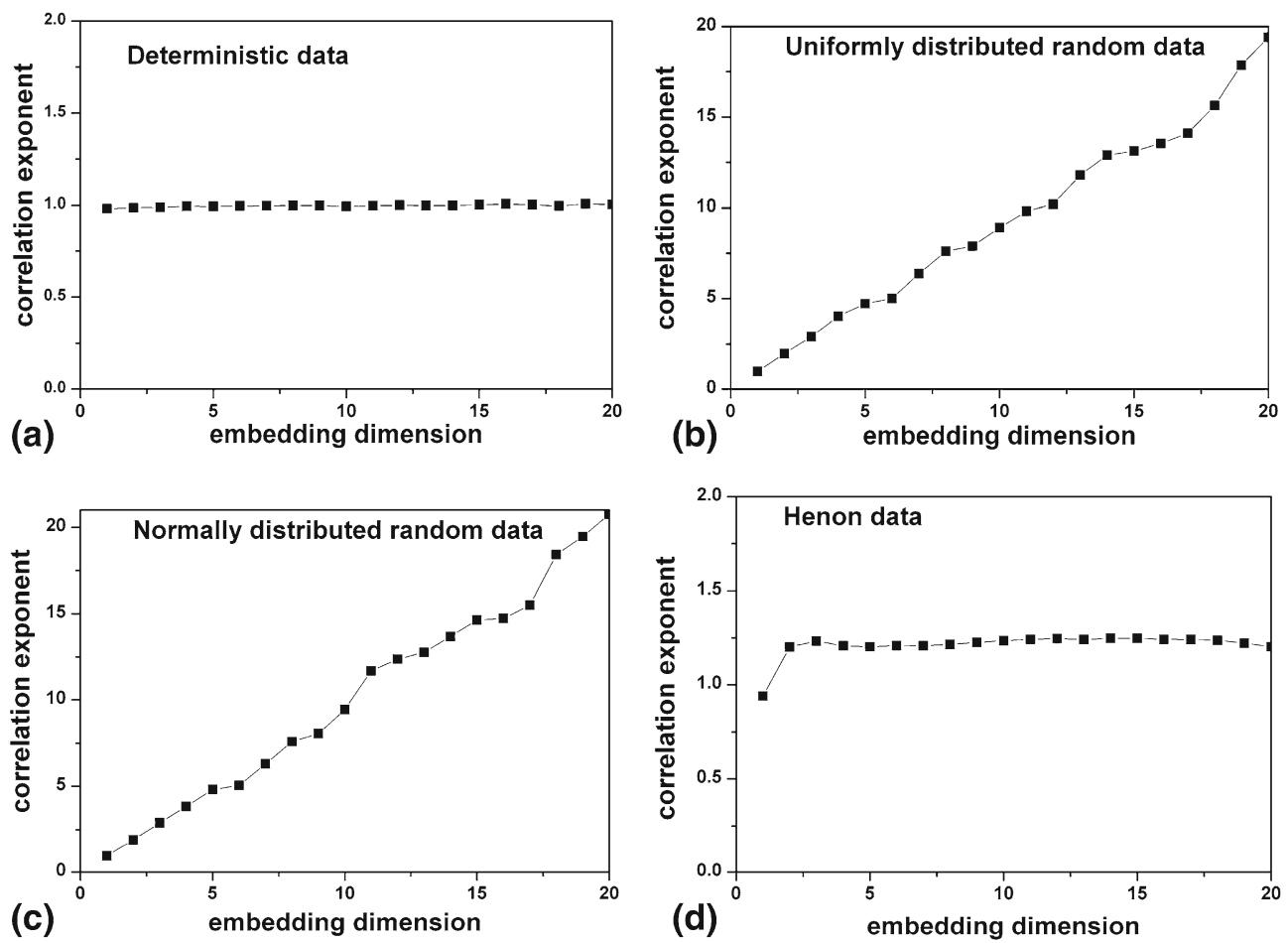

Figure 5. Embedding dimension vs. correlation exponent plot of the time series under behavioural study. 
The saturation of correlations exponents at 1.2 after an initial increase represents chaotic nature of Henon data (refer figure 5d). The nearest whole number integer of 2 will be the number of dimensions required for prediction models to obtain the best results. Thus, it may be concluded that the Grassberger-Procaccia algorithm can be used to study the behaviour of a time series.

\subsection{Behavioural study of Koyna station daily rainfall data}

After verifying the applicability of correlation dimension method to study the behaviour of a time series, it is applied to a real life rainfall data. Forty seven years of daily rainfall data from $1^{s t}$ January 1961 to $31^{s t}$ December 2007 observed at Koyna dam is used for the chaotic analysis. The statistics of rainfall data is given in table 1 . The time series plot, phase space plot, $\log r$ vs. $\log \mathrm{Cr}$ plot and finally the embedding dimension vs. correlation exponent plot of observed rainfall data observed at Koyna raingauge station is given in figures $6 \mathrm{a}$ to $\mathrm{d}$. The time series plot of the observed rainfall shows the intermittent nature of the rainfall. There is rainfall only during monsoon periods and rest of the period it is zero. From figure 6a it is clear that the time series plot is not continuous as it has large number of zero values. Around $70 \%$ of the data contains zero values as rainfall happens only in five months from June to October. The phase space plot
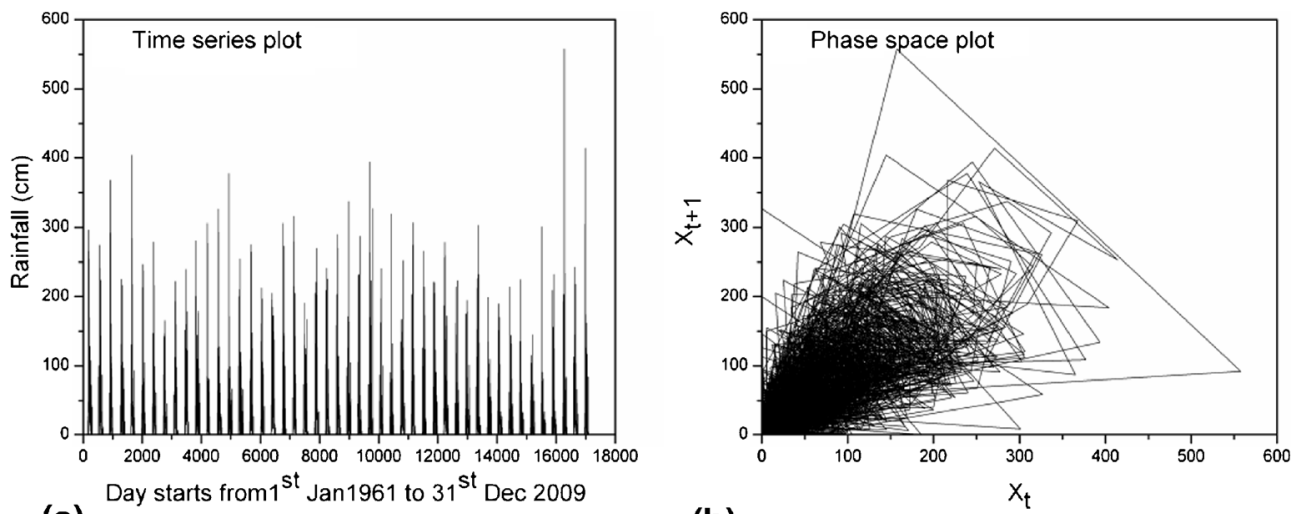

(a)

(b)
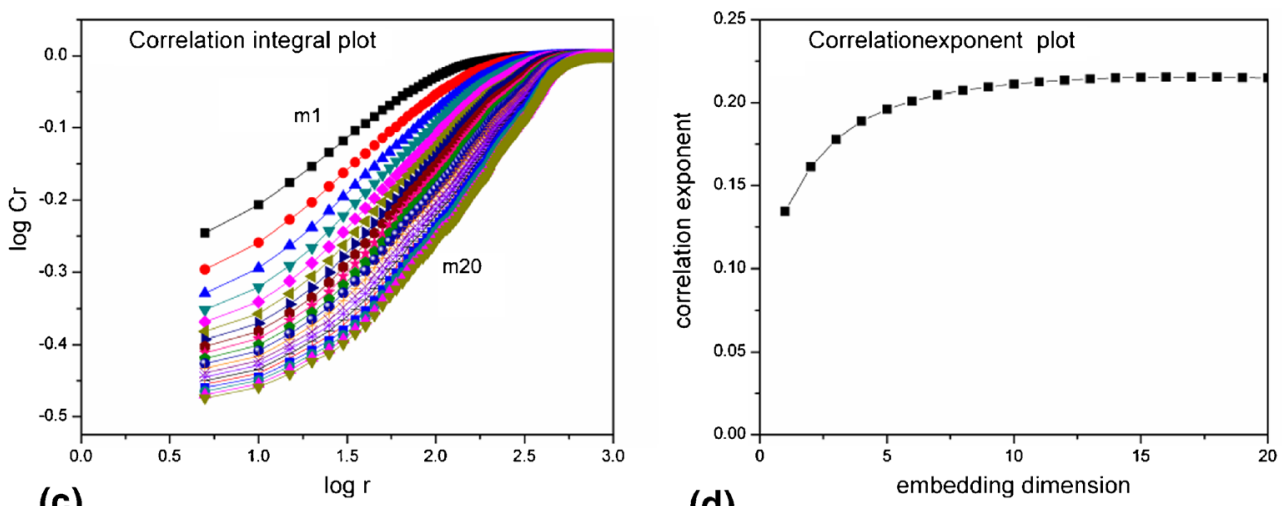

(d)

Figure 6. Chaotic behavioural results of the observed daily rainfall at Koyna rain gauge station. 
of rainfall is given in figure 6b. The phase space plot of rainfall shows a similarity to normally distributed random numbers, which is stochastic in nature. The only difference is that majority of points are having trajectory towards the origin as the rainfall data has higher percentage of zero values and hence the attractor is concentrated towards origin. From the correlation integral plot (log r vs. $\log \mathrm{Cr}$ plot) of rainfall given in figure $6 \mathrm{c}$ it is clear that at lower range of ' $r$ ' the lines are parallel to each other from which it is difficult to estimate the slope whereas at higher range of ' $r$ ' the plot is almost similar to Henon data which gives an idea that the behaviour of rainfall data may be chaotic. The correlation exponent variation with embedding dimension ranging from one to twenty is given in figure $6 \mathrm{~d}$. The correlation exponents started saturating at 0.38 which confirms the behaviour of rainfall as chaotic and hence the minimum embedding dimension to be considered for the prediction modelling may be one (the next nearest integer of 0.38 ). But this underestimation of correlation dimensions may be due to higher percentage of zero values in rainfall data (Sivakumar 2001). However, the deviation of correlation exponents from one dimension to the next is less than $10 \%$ from an embedding dimension of five which is considered as the optimum embedding dimension to be taken for modelling. Thus, in the present case to predict the daily rainfall data the number of inputs may be five.
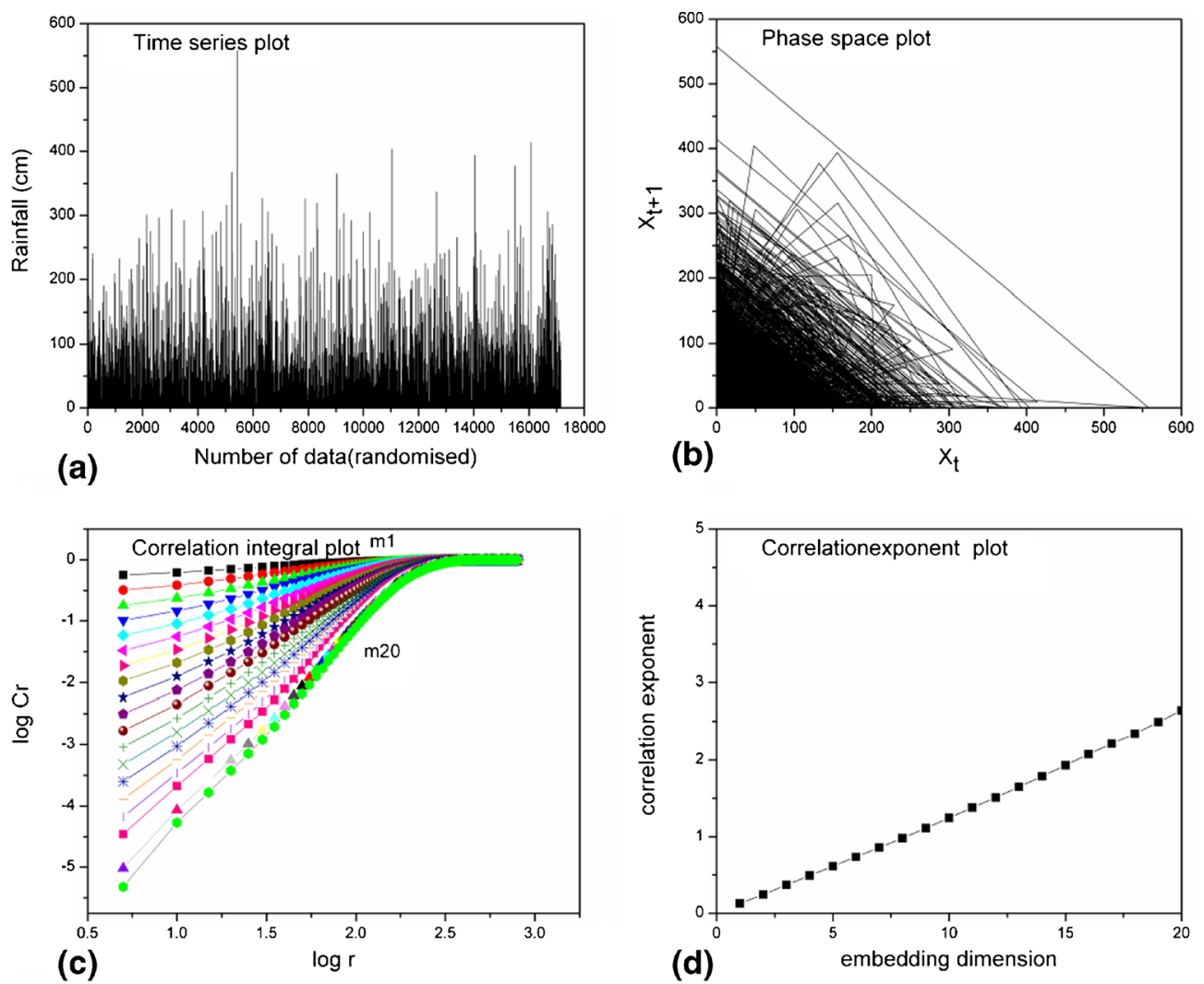

Figure 7. Chaotic behavioural results of the surrogate rainfall data. 
4.4a Phase randomisation: The saturation of correlation exponents may be due to the inherent linear correlation structure which can be verified by analysing surrogate data generated by phase randomisation (Theiler et al 1992). The time series plot, phase space plot, log r vs. log Cr plot and finally the embedding dimension vs. correlation exponent plot of surrogate rainfall data is given in figures $7 \mathrm{a}$ to $\mathrm{d}$. The continuous zero values are shuffled by randomisation and hence the time series plot of surrogate data is entirely different from observed rainfall data (refer figures 6a and 7a). The geometry of phase space is different from the observed data where as the outer surface of the phase space diagram is exactly same in both the cased of observed and surrogate data. The correlation integral plot ( $\log r$ vs. $\log \mathrm{Cr}$ ) and correlation exponent plot (embedding dimension vs. correlation exponent plot) is entirely different in both the cases. The surrogate data shows the behaviour as stochastic and hence the null hypothesis is eliminated which further confirms the behaviour of rainfall as chaotic. The correlation exponents in this case are more than four which may be due to the distribution of long continuous zero values by randomisation.

\subsection{Behavioural study using false nearest neighbour method}

In the ideal case, which is for a pure chaotic data, the percentage of false neighbours will drop to zero after increasing the embedding dimension. In this case of chaotic data, once the percentage of false neighbours drops it will never increase further with higher embedding dimension. The dimension at which it falls to zero will be the optimum embedding dimension in the ideal case. The FNN plots of the time series used in the present study is given in figure 8 . The chaotic behaviour of Henon data is very clear from FNN plot as the percentage of false neighbours has dropped to zero. In case of random data percentage of false neighbours has dropped to around $20 \%$ but it again increases and reached $100 \%$ which is the indication of stochastic nature. In the case of rainfall series the percentage is not dropping to zero and increasing which creates an

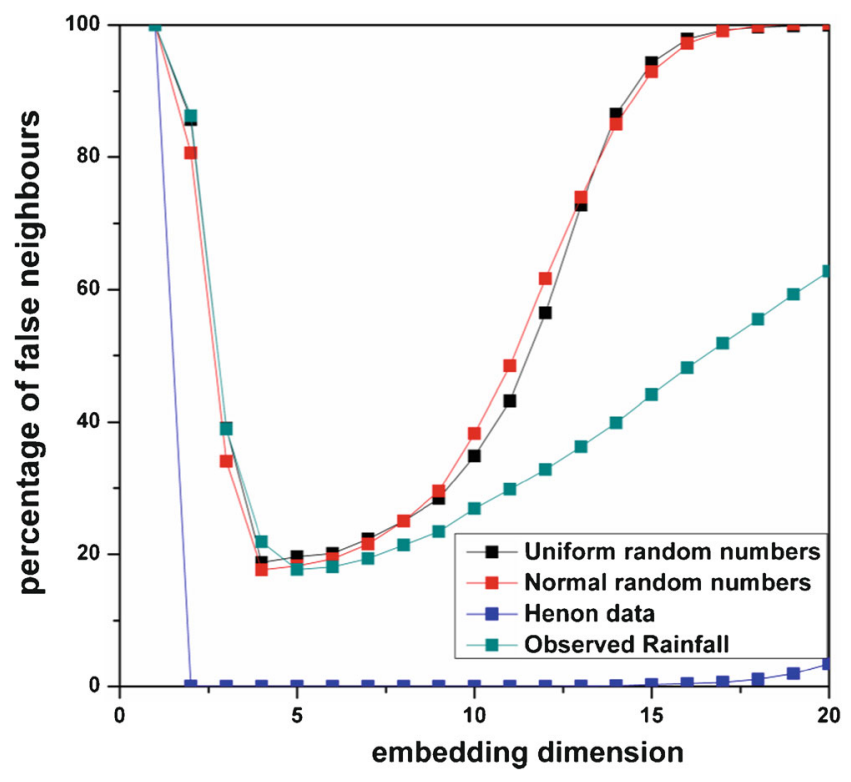

Figure 8. FNN plots of time series under behavioural study. 
ambiguity to determine the behaviour, but the increase is not $100 \%$ indicating that the series is not stochastic, thus probably may be a chaotic series. Thus, the optimum embedding dimension can be fixed at the dimension at which the percentage of false neighbour is least. In the present case of rainfall series, the dimension using FNN method is five. On comparing the results of CDM and FNN applied for time series, both the methods have resulted in similar behaviour. But, in case of rainfall data, the CDM method has underestimated the correlation dimension than FNN, but the optimum value of embedding dimension resulted from CDM method is five, same as that resulted from FNN. The underestimation of correlation dimension in case of CDM may be due to the longer number of zero values in the time series. Thus, one should be careful in using CDM for chaotic analysis of time series having longer length of zero values.

\section{Conclusions}

Correlation dimension method is an efficient method for classification of data based on its behaviour. In the present case, five sets of time series data are analyzed using correlation dimension method for classification purpose. The embedding dimension vs. correlation exponent plot for all these five data sets helps to fix the behaviour of these time series. Based on the results from correlation dimension method, the behaviour of natural numbers turns out to be deterministic whereas the behaviour of uniform and normal random numbers is stochastic irrespective of its type of distribution. The behaviour of Henon data is chaotic from correlation dimension method. In addition to the behaviour classification the method identifies the number of dominant variables for the further prediction models. The results obtained from the analysis of series with known behaviour arrive at a conclusion that correlation dimension method can be used for the behaviour study of real life data also. The correlation dimension method applied to real life rainfall data brings out the behaviour of rainfall at Koyna station is chaotic as the correlation exponent saturates. However, correlation dimension is underestimated in the present study which may be due to higher percentage of zero values in rainfall. The under estimation of correlation dimension is solved by FNN in case of rainfall data. However, FNN results are helpful to fix the optimum embedding dimension once the behaviour is known. The behaviour is further confirmed by phase randomisation which again supported the results arrived from CDM and FNN. The optimum dimension required for modelling rainfall data at Koyna region is turned out to be five in the present study.

\section{References}

Abarbanel H D I, Brown R, Sidorowich J J and Tsimring L S 1993 The analysis of observed chaotic data in physical systems. Reviews of Modern Phys., 65(4): 1331-1392

Adhikari R and Agrawal R K 2013 An introductory study on time series modelling and forecasting. Germany: LAP LAMBERT Academic Publishing

ASCE (American Society of Civil Engineers) Task committee on application of the neural networks in hydrology 2000a Artificial neural networks in hydrology I: Preliminary concepts. J. Hydrol. Eng. ASCE, 5(2): $115-123$

ASCE (American Society of Civil Engineers) Task committee on application of the neural networks in hydrology 2000b Artificial neural networks in hydrology II: Hydrologic applications. J. Hydrol. Eng. ASCE, 5(2): 124-137

Box G E P and Jenkins G M 1976 Time series analysis forecasting and control, 2nd edn. Holden day, San Francisco, CA: Colorado State University, Fort Collins, Co 
Box G E P, Jenkins G M and Reinsel G C 1994 Time series analysis: Forecasting and control. Delhi: Pearson Education

Brameier M and Banzhaf W 2007 Linear genetic programming. New York: Springer Science

Brockwell P J and Davis R A 2002 Introduction to time series and forecasting, 2nd ed. New York: Springer-Verlag

Cao L 1997 Practical method for determining the minimum embedding dimension of a scalar time series. Phys. D, 110: 43-50

Chang F J, Chang L C and Huang H L 2002 Real-time recurrent learning neural network for stream-flow forecasting. Hydrol. Process, 16(13): 2577-2588

Chatfield C 1996 The analysis of time series - an introduction, 5th ed. London, UK: Chapman and Hall

De Vos N J and Rientjes 2005 Constraints of artificial neural networks for rainfall-runoff modelling: tradeoffs in hydrological state representation and model evaluation. Hydrol. Earth Syst. Sci., 9: 111-126

Dhanya C T and Nagesh Kumar D 2010 Nonlinear ensemble prediction of chaotic daily rainfall. Advances in Water Resources 33(3): 327-347

Dhanya C T and Kumar D N 2011 Predictive uncertainty of chaotic dailystreamflow using ensemble wavelet networks approach. Water Resources Research 47(6): 1-28

Fraser A and Swinney H 1986 Independent coordinates for strange attractors from mutual information. Physical Review A, 33(2): 1134-1140

Garg V and Jothiprakash V 2010 Reservoir trap efficiency estimation using artificial neural networks and genetic programming. J. Hydrol. Eng. ASCE 12(15): 1001-1015

Govindaraju R S 2002 Preliminary concepts in stochastic processes, in Stochastic methods in subsurface contaminant hydrology, R S Govindaraju (ed.), New York: ASCE Press

Govindaraju R S and Rao A R 2000 Artificial neural networks in hydrology. Dordrecht, The Netherlands: Kluwer Academic

Grassberger P and Procaccia I 1983a Characterization of strange attractors. Physical Review Lett., 50(5): 346-349

Grassberger P and Procaccia I 1983b Measuring the strangeness of strange attractors. Physical D: Nonlinear Phenomena, 9(1-2): 189-208

Guven A 2009 Linear genetic programming for time-series modeling of daily flow rate. J. Earth Sys. Sci. 118(2): 137-146

Hamzacebi C 2008 Improving artificial neural networks' performance in seasonal time series forecasting. Information Sci., 178: 4550-4559

Harms A A and Campbell T H 1967 An extension to the Thomas-Fiering model for the sequential generation of stream flow. Water Resources Research 3(3): 653-661

Harvey H C 1981 Time series models. New York, NY, USA: Halstead Press

Hense A 1987 On the possible existence of a strange attractor for the southern oscillation. Beitrage of Phys. Atmosphere 60(1): 34-47

Hipel K W and McLeod A I 1994 Time series modeling of water resources and environmental systems. Amsterdam: Elsevier

Islam M N and Sivakumar B 2002 Characterisation of runoff dynamics: A nonlinear dynamical view. Advances in Water Resources, 25: 179-190

Jayawardena A W and Lai F 1994 Analysis and prediction of chaos in rainfall and stream flow time series. J. Hydrol. 153: 23-52

Jothiprakash V, Magar R and Sunil K 2007 Rainfall-runoff modelling using Linear Regression- a case study of an intermittent river. J. Indian Association Environm. Management 34(3): 125-131

Jothiprakash V and Magar R B 2009 Soft computing tools in rainfall-runoff modeling. ISH J. Hydraul. Eng. 15(SP.1): 84-96

Jothiprakash V and Kote A 2011 Effect of pruning and smoothing while using M5 model tree technique for reservoir inflow prediction. J. Hydraul. Eng. 16(7): 563-574

Jothiprakash V and Magar R B 2012 Multi-time-step ahead daily and hourly intermittent reservoir inflow prediction by artificial intelligent technique using lumped and distributed data. J. Hydrol. 450-451: 293-307 
Jothiprakash V and Fathima T A 2013a Chaotic analysis of reservoir inflow series- a case study on Koyna reservoir inflow. J. Institute of Eng. (India), 94(2): 89-97

Jothiprakash V and Fathima T A 2013b Chaotic analysis of daily rainfall series in Koyna reservoir catchment area. Stochastic Environmental Research and Risk Assessment, 27(6): 1371-1381

Kantz H and Schreiber T 1997 Nonlinear time series analysis. Cambridge, UK: Cambridge University Press

Kantz H and Schreiber T 2003 Nonlinear time series analysis, 2nd ed. Cambridge, UK: Cambridge University Press

Kavvas M 2003 Nonlinear Hydrologic processes: conservation equations for determining their means and probability distributions. J. Hydrol. Eng. 8(2): 44-53

Kennel M, Brown R and Abarbanel H 1992 Determining embedding dimension for phase-space reconstruction using a geometrical construction. Physical Review A, 45(6): 3403-3411

Khatibi R, Ghorbani M A, Aalami M T, Kocak K, Makarynskyy O, Makarynska D and Aalinezhad D 2011 Dynamics of hourly sea level at Hillary Boat Harbour, Western Australia: a chaos theory perspective. $J$. Ocean Dynamics 61(11): 1797-1807

Khu S T, Liong S, Vladan B Henrik M and Muttil N 2001 Genetic programming and its application in real-time runoff forecasting. J. Am. Water Resources Association 37(2): 439-451

Klemes V 1978 Physically based stochastic hydrologic analysis. Adv. Hydrosci. 11: 285-352

Kumar S 2004 Neural networks, a classroom approach. India: Tata McGraw-Hill Publishing Company Limited

Magar R B and Jothiprakash V 2011 Intermittent reservoir daily-inflow prediction using lumped and distributed data multi-linear regression models. J. Earth Sys. Sci. 120(6): 1067-1084

Mitasova H and Mitas L 1998 Process modeling and simulations NCGIA core curriculum in GIScience, http://www.ncgia.ucsb.edu/giscc/units/u130/u130.html

Pal M and Deswal S 2009 M5 model trees based modelling of reference evapotranspiration. Hydrol. Process. 23: 1437-1443

Pankratz A 1983 Forecasting with Univariate Box-Jenkins models: concepts and cases. New York: John Wiley

Porporato A and Ridolfi L 1997 Nonlinear analysis of river flow time sequences. Water Resources Research, 33(6): 1353-1367

Quinlan J R 1992 Learning with continuous classes. Proceedings of the Australian Joint Conference on Artificial Intelligence World Scientific, Singapore, pp. 343-348

Ramasubramanian V 2007 Time Series Analysis I.A.S.R.I., Library Avenue, New Delhi

Salas J D and Smith R A 1981 Physical basis of stochastic models of annual flows. Water Resources Research 17(2): 428-430

Sano M and Sawada Y 1985 Measurement of the lyapunov spectrum from a chaotic time series. Physical Review Letters, 55(10): 1082-1085

Shivamoggi B K 1997 Nonlinear dynamics and chaotic phenomena: An introduction. Netherland: Kluwer Academic Publishers

Sivakumar B 2000 Chaos theory in hydrology: Important issues and interpretations. J. Hydrol. 227: 1-20

Sivakumar B 2001 Rainfall dynamics at different temporal scales: A chaotic perspective. Hydrol. and Earth Sys. Sci., 5(4): 645-651

Sivakumar B and Jayawardena A W 2002 An investigation of the presence of low-dimensional chaotic behavior in the sediment transport phenomenon. Hydrol. Sci. J., 47(3): 405-16

Sivakumar B, Liong S-Y and Liaw C-Y 1998 Evidence of chaotic behavior in Singapore rainfall. J. Am. Water Resources Association 34(2): 301-310

Sivakumar B, Jayawardena A W and Fernando T M G H 2002 River flow forecasting: Use of phase-space reconstruction and artificial neural networks approaches. J. Hydrol. 265(1-4): 225-245

Sivakumar B, Jayawardena A W and Li W K 2007 Hydrologic complexity and classification: a simple data reconstruction approach. Hydrol. Process. 21: 2713-2728

Solomatine D P and Dulal K N 2003 Model trees as an alternative to neural networks in rainfall-runoff modeling. Hydrol. Sci. J. 48(3): 399-411 
Strogatz S H 1994 Nonlinear dynamics and chaos. Addison-Wesley, United States of America: Perseus Books Publishing

Takens F 1981 Detecting strange attractors in turbulence. In: Dynamical Systems and Turbulence Lecture Notes in Mathematics 898, D A Rand, LS Young (eds.), Berlin Springer, pp. 366-381

Theiler J, Eubank S, Longtin A, Galdrikian B and Farmer J D 1992 Testing for nonlinearity in time series: the method of surrogate data. Phys. D 58: 77-94

Thirumalaiah K and Deo M C 2000 Hydrological forecasting using neural networks. J. Hydrol. Eng. ASCE 5(2): 180-189

Tokar A S and Johnson P A 1999 Rainfall-runoff modeling networks. J. Hydrol. Eng. ASCE, 4(3): 232-239

Tong H 1990 Non-Linear time series: A dynamical system approach. Oxford: Oxford University Press

Williams P 1997 Chaos theory Tamed US Geological Survey (Ret.) Joseph Henry Press, Washington

Wu J S, Han J, Annambhotla S and Bryant S 2005 Artificial neural networks for forecasting watershed runoff and stream flows. J. Hydrol. Engi. ASCE 10(3): 216-222

Zhang G 1998 Forecasting with artificial neural networks: The state of art. Int. J. Forecasting 14: 35-62 